Supporting Information for

\title{
Effect of Hydrogen Bond on Photochemical Synthesis of Silver Nanoparticles
}

\author{
Feng-jiao Zhao ${ }^{\dagger}$, Lei Liu ${ }^{\dagger,}$, Yang Yang ${ }^{\dagger,}$, Da-li Xu', Rui-ling Zhang ${ }^{\dagger}$,
}

Guang-hua Ren", Pan-wang Zhou', Ke-li Han ${ }^{\dagger}$

†State Key Laboratory of Molecular Reaction Dynamics, Dalian Institute of Chemical Physics, Chinese Academy of Sciences, Dalian 116023, China

${ }^{\S}$ State Key Laboratory of Fine Chemicals, Dalian University of Technology, Dalian 116024, China

${ }^{*}$ Key Laboratory of Chemical Lasers, Dalian Institute of Chemical Physics, Chinese Academy of Sciences, Dalian 116023, China

Table of contents

1. Laser energy in experiment of transient absorption spectrum

2. Lifetimes of ketyl radicals

3. Centrifugation Method 
1. Laser energy in experiment of transient absorption spectrum

In our transient absorption spectrum experiment, for an appropriate laser energy we measured the correlation of absorbance intensity and laser pulse energy (laser wavelength is $355 \mathrm{~nm}$ and the absorbance wavelength is $525 \mathrm{~nm}$ ). Sample solution is $5 \mathrm{mM}$ benzophenone $(\mathrm{BP})$ in acetonitrile $(\mathrm{ACN})$. We found that there existed a threshold for laser energy which is about $60 \mathrm{~mJ}$. We set $25 \mathrm{~mJ}$ as our laser pulse energy in this work.

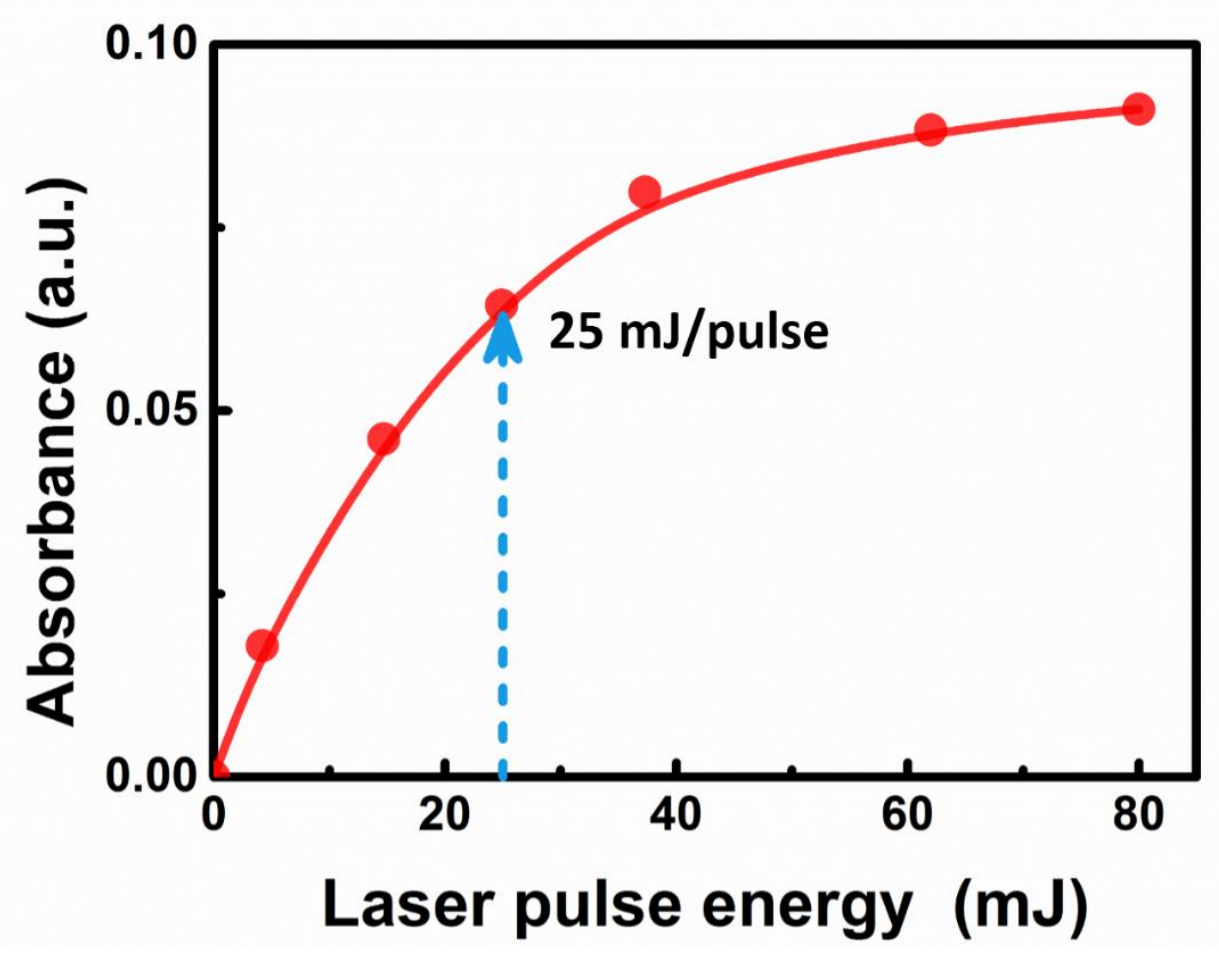

Figure S1. Correlation of absorbance intensity and laser pulse energy. $25 \mathrm{~mJ} / \mathrm{pulse}$ is set as laser energy in this work. 


\section{Lifetimes of ketyl radicals}

From the transient absorbance spectra in Figure 2, we can find that BP $\mathrm{T}_{1}$ and ketyl radicals have different lifetimes in different systems. For the purpose of studying the correlation between efficiency of $\mathrm{Ag}^{0}$ nanoparticles photosynthesis and lifetimes of ketyl radicals, exponential fitting analysis has been performed and the fitting results are displayed in Figure S2.

It is clear that fitting curves (red lines) agree fairly well with raw data (blue dots). For the lifetime of BP $\mathrm{T}_{1}$, due to all the absorbance in Figure 2(a) comes from $\mathrm{BP} \mathrm{T}_{1}$, we select the strongest absorbance dynamic trace at $530 \mathrm{~nm}$ for monoexponential lifetime

fitting. The lifetime of BP $\mathrm{T}_{1}$ was estimated to be $1.232 \pm 0.030 \mu$ s with the adjusted $\mathrm{R}^{2}$ of 0.940 .

For ketyl radical, it is widely accepted that its characteristic absorbance peak is at around 530nm. Since the BP $\mathrm{T}_{1}$ was still able to be detected in BP-ET system, the absorbance peak at 530nm should be partly involved in $\mathrm{BP}_{1}$. Therefore, absorbance at $530 \mathrm{~nm}$ in Figure 2(b) should be attributed to the mixture signal of $\mathrm{BP} \mathrm{T}_{1}$ and ketyl radical complex. For these reasons, biexponential fitting method is reasonable and was performed at 530nm. Two fitting lifetimes were obtained of $0.275 \pm 0.041 \mu$ s and $3.833 \pm 0.182 \mu$ s with the adjusted $\mathrm{R}^{2}$ of 0.916 . As the hydrogen abstracting quenching of $\mathrm{BP} \mathrm{T}_{1}$ by $\mathrm{ET}$, the lifetime of $\mathrm{BP} \mathrm{T}_{1}$ in this system should be shorter than that in the system of BP alone. Also, it has been reported that hydrogen-bonded biradical 
complex has a longer lifetime than triplet. ${ }^{1}$ Thus, in the system of BP-ET, lifetimes of $\mathrm{BP}_{1}$ and ketyl radical should be $0.275 \pm 0.041 \mu$ s and $3.833 \pm 0.182 \mu \mathrm{s}$, respectively. Meanwhile, for keytl radical in BP-CHD system, according to Scheme 1(c), the single absorbance at 545nm (Figure 2(c)) was assigned to the mixture of ketyl radical and the cyclohexadienyl radical. This spectrum result agrees well with the early works. ${ }^{2,3}$ Thus, biexponential fitting method was again used at 545nm. The fitting lifetimes were $4.063 \pm 0.101 \mu \mathrm{s}$ and $35.716 \pm 0.473 \mu \mathrm{s}$ with the adjusted $\mathrm{R}^{2}$ of 0.996 . To distinguish the lifetimes of two kinds of radicals, we seek for the established works and get inspired. As reported by limori et al., CHD plays both roles of $\mathrm{H}$ donor and micelle. Particularly, cyclohexadienyl radical escapes from the micelle clusters immediately after the hydrogen abstraction meanwhile the ketyl radical survives in the micelle clusters. ${ }^{4,5}$ Because the radicals outside the micelle are quenched more easily with other molecules, we prefer to ascribe the relatively longer lifetime to ketyl radical and the shorter one to cyclohexadienyl radical. Consequently, lifetimes of ketyl radical and cyclohexadienyl radical are $35.716 \pm 0.473 \mu$ s and $4.063 \pm 0.101 \mu$ s, respectively. This lifetime of $4.06288 \pm 0.10051 \mu$ s for cyclohexadienyl radical agrees well with other works. ${ }^{5-7}$

Fitting results are summarized and shown in Table S1. It displays that the lifetimes of ketyl radical in BP-ET and BP-CHD systems are 3.833 $\pm 0.182 \mu$ s and $35.716 \pm 0.473$ $\mu s$, respectively. Therefore, the efficiency of hydrogen abstraction reaction is proportional to product (ketyl radical) lifetime. Moreover, as shown in Figure 5-8, 
ketyl radical complex exhibits stronger reductibility than ketyl radical. The lifetime result agrees with it for the shorter-lived radical has higher reaction activity.
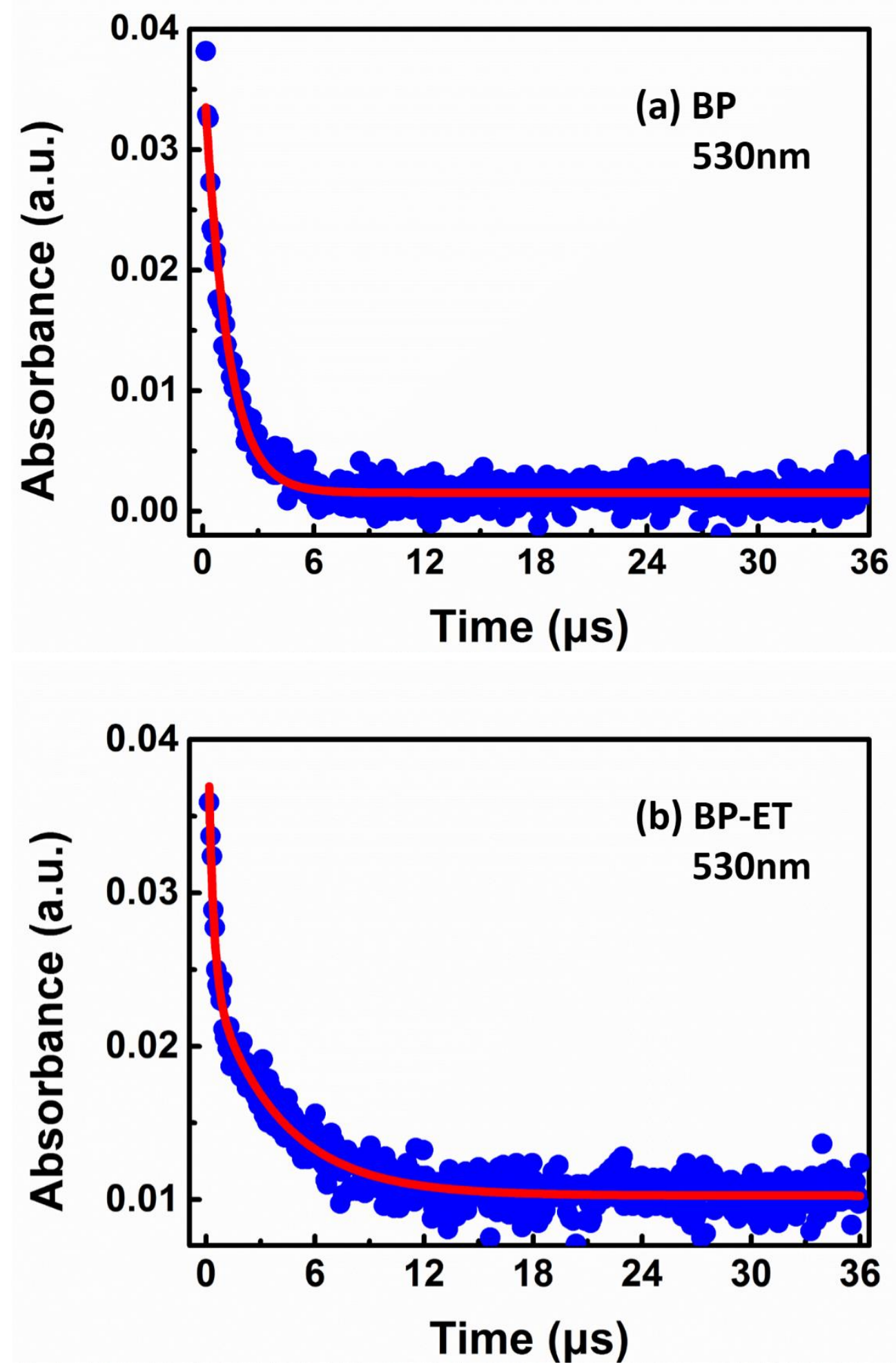


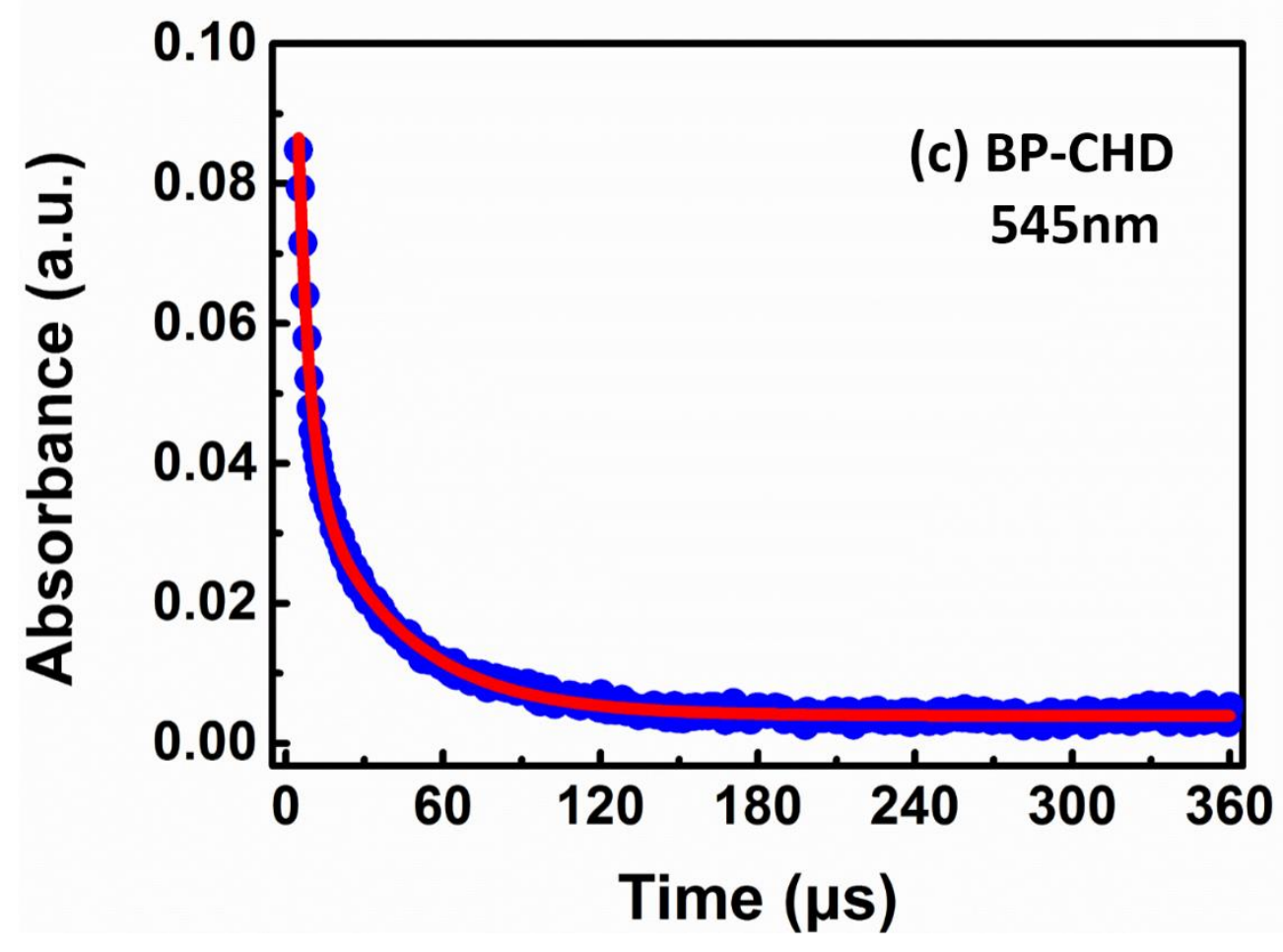

Figure S2. Transient absorbance of BP $(5 \mathrm{mM})$ with and without $\mathrm{H}$ donors $(50 \mathrm{mM})$ in deaerated acetonitrile $(\mathrm{ACN})$ at different wavelengths excited by $355 \mathrm{~nm}$ laser pulses at room temperature. The systems measured and wavelengths of absorption are: (a) BP, at 530nm; (b) BP with ET, at 530nm; (c)BP with CHD, at 545nm. The blue dots were the original data and the red lines were the fitting curves.

Table S1. Lifetime of BP $\mathrm{T}_{1}$ and radicals in different systems. '-' means there is no such kind of species or the lifetime is too short to be detected.

\begin{tabular}{|l|l|l|l|}
\hline & BP T1 & Ketyl radical & CHD radical \\
\hline BP & $1.232 \pm 0.030 \mu \mathrm{s}$ & - & - \\
\hline BP+ET & $0.275 \pm 0.041 \mu \mathrm{s}$ & $3.833 \pm 0.182 \mu \mathrm{s}$ & - \\
\hline BP+CHD & - & $35.716 \pm 0.473 \mu \mathrm{s}$ & $4.063 \pm 0.101 \mu \mathrm{s}$ \\
\hline
\end{tabular}




\section{Centrifugation Method}

Before the measurement of Inductively Coupled Plasma-Optical Emission Spectroscopy (ICP-OES), we conducted centrifugation to eliminate the $\mathrm{Ag}^{0}$ from solution. In order to test the effectiveness of centrifugation in our experiment, we performed the UV/VIS absorbance spectrum of $\mathrm{Ag}^{0}$ NPs before and after centrifugation in BP and ET system (shown in Figure S3). Before irradiation, there is no absorbance at 400nm (Grey line in Figure S3). After 30min of laser irradiation at $355 \mathrm{~nm}, \mathrm{Ag}^{0}$ NPs have been produced and strong absorbance around 400nm appears (Red line in Figure S3). It is clear that after 40min of centrifugation at the revolution speed of $15000 \mathrm{r} / \mathrm{min}$, the absorbance from $350 \mathrm{~nm}$ to $600 \mathrm{~nm}$ is decreasing to zero (Blue line in Figure S3) which means $\mathrm{Ag}^{0}$ has been centrifuged and removed completely from solution. 


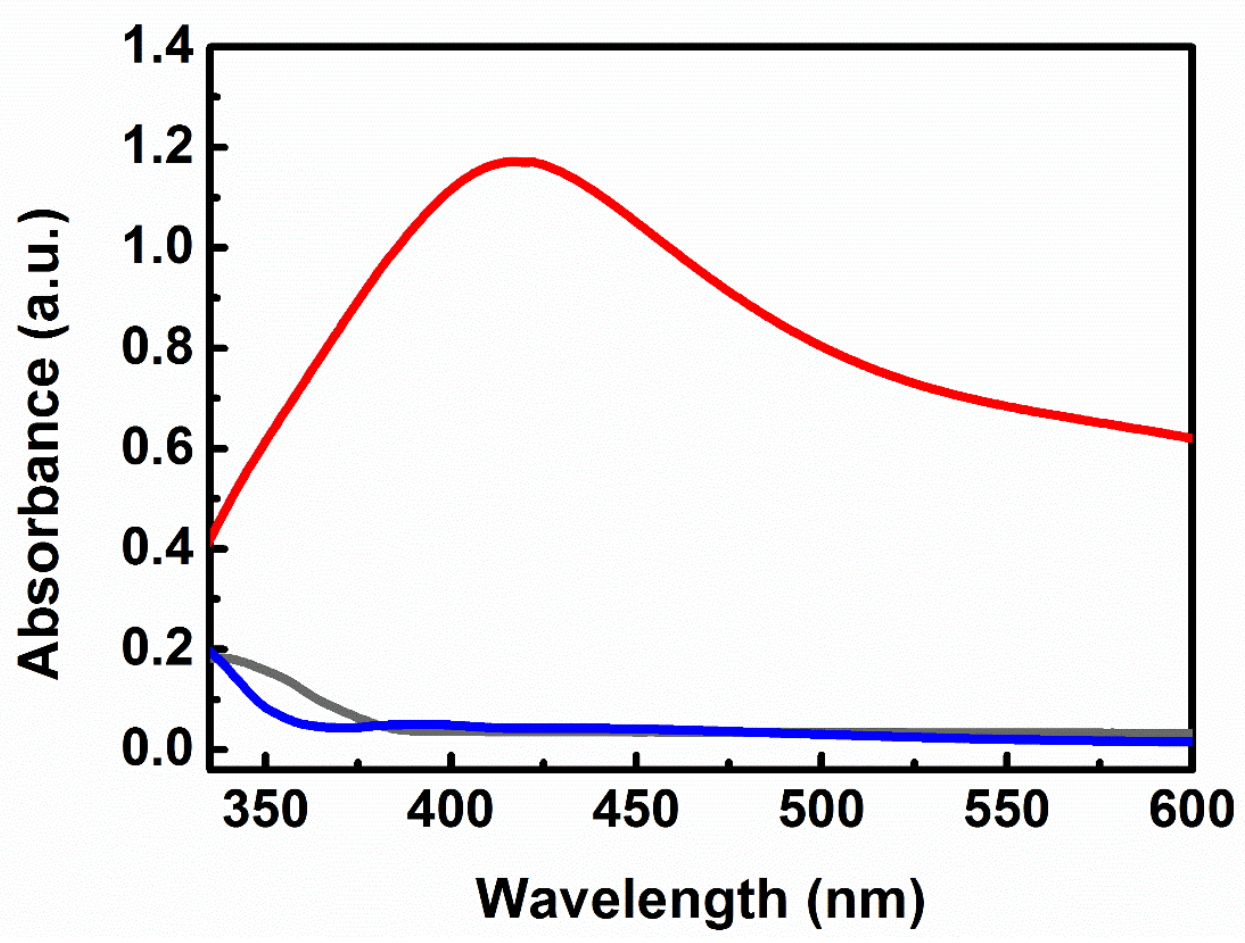

Figure S3. Absorbance spectra of $\mathrm{Ag}^{0} \mathrm{NPs}$ in solution. (a) Grey line: before irradiation; (b) Red line: after irradiation (30min at 3Hz); (c) Blue line: after centrifugation of $40 \mathrm{~min}$ at the revolution speed of $15000 \mathrm{r} / \mathrm{min}$.

Reference

[1]William J. Leigh, Edward C. Lathioor and Michael J. St. Pierre,

J.Am.Chem.Soc.,1996,118,12337-12348

[2]H. BAUMANN, C. MERCKEL, H.-J. TIMPE, Chem. Phys. Lett. ,1984, Vol.103,No.6 
[3]Linda J. Johnston, D. J. hugnot, VGronique Wintgen and J. C. Scaiano, J. Am.

Chem. SOC., 1988, 110, 518-524

[4]Yutaka Shiga, Mitsuo Koshi, Kenichi Tonokura, Chem Phys Lett, 470, 2009, 3538.

[5]Toshifumi Iimori, Hironao Tanaka, Akio Kawai, Kinichi Obi, Chemical Physics Letters, 309, 1999, 351-356

[6]Myran C. Sauer, Jr., and Inder Mani, Journal of Physical Chemistry, Vol.72, No.11, 1968, 3856-3862

[7]Myran C. Sauer, Jr., and Barry Ward, Journal of Physical Chemistry, Vol.71, No.12, 1967, 3971-3983 